\title{
The Effect of Signal-signal and Signal-idler Modes on Squeezing and Entanglement Properties of Two-mode Light
}

\author{
Misrak Getahun \\ Department of Physics, Hawassa University, P. O. Box 05, Awassa, Ethiopia
}

\begin{abstract}
Squeezed and entangled two-mode light can be generated by the combination of degenerate and nondegenerate parametric oscillators. The correlated signal-idler modes are not only enhance the degree of quadrature squeezing and entanglement properties of the two-mode light but they are also the cause of these properties. But the effect of signal-signal modes are to enhance the degree of quadrature squeezing and to reduce the entanglement property of the two-mode light.
\end{abstract}

Keywords: signal-signal modes, signal-idler modes, quadrature squeezing, entanglement

DOI: $10.7176 /$ APTA/78-02

Publication date:June $30^{\text {th }} 2019$

\section{Introduction}

The interaction of radiation with a nonlinear crystal leads to the production of a radiation with nonclassical features such as quadrature squeezing and entangled two-mode light. In a down single photon conversion process, each pump photon to be annihilated inside the cavity and produce a pair signal-signal degenerate photons or signal-idler nondegenerate photons of the cavity modes [1] - [14]. It has been predicted that a parametric oscillator produces light with quadrature squeezing [5], [6] and entangled modes [7], [8] that is due to the quantum correlation between the two modes.

A squeezed state of light features reduces quantum noise in one quadrature component below the standard quantum limit (vacuum or coherent state). The minimum uncertainty of the product of the two quadrature components must be preserved by enhancing quantum noise in other quadrature component. Squeezed light has application in optical communication and high sensitively quantum detectors.

It is believed that entanglement is the bases for emerging technologies as quantum computation [15] and quantum cryptography [16]. Recently, much attention has given to the generation and detection of continuousvariable entanglement for quantum information. The efficiency of the quantum information processing highly depends on the degree of entanglement. Hence, it is desirable to generate strongly entangled continuous-variable state. Schemes for generating continuous-variable entanglement have been proposed and realized in nondegenerate parametric amplifiers [14], [19].

In this paper we propose a system made up of a combination of subharmonic degenerate and nondegenerate parametric oscillators. We wish to investigate the squeezing and entanglement properties of a two-mode light produced by the system under consideration, employing the steady state solutions of the cavity mode operators and the correlation properties of the noise operators.

\section{The Cavity Operators}

We consider a cavity contains three nonlinear crystals in which the pump modes are down converted into a pair of highly correlated signal-signal and signal-idle modes. The two crystals are used to produce signal-signal modes $\hat{a}_{1}-\hat{a}_{1}$ and $\hat{a}_{2}-\hat{a}_{2}$ and the other one is used to generate signal-idle modes $\hat{a}_{1}-\hat{a}_{2}$ as shown in FIG. 1.

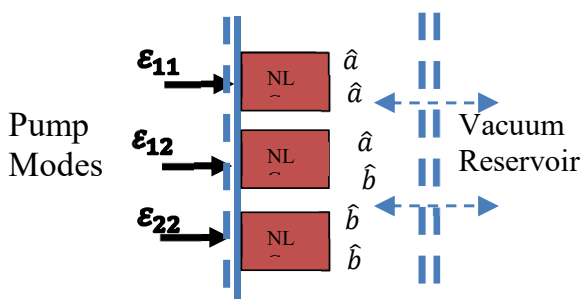

FIG. 1 Schematic representation of combined subharmonic parametric oscillators.

\subsection{Master Equation}

With the pump modes treated classically, the subharmonic parametric process can be described in the interaction picture by the Hamiltonian

$$
\hat{H}_{s}=\frac{i}{2} \sum_{j, k}^{2,2} \varepsilon_{j k}\left(\hat{a}_{j}^{\dagger} \hat{a}_{k}^{\dagger}-\hat{a}_{j} \hat{a}_{k}\right),
$$


in which $\varepsilon_{j k}=\varepsilon_{k j}=\lambda_{j k} \beta_{j k}$ considered to be real, are the amplitudes proportional to the pump modes, with $\beta_{j k}$ and $\lambda_{j k}$ being the c-number variable for the pump modes and the coupling constants for subharmonic generation, respectively and $j, k=1,2$. The equation of motion for the density operator corresponding to Eq. (1) with the cavity modes being coupled to vacuum reservoir can be expressed in the form

$$
\frac{d \hat{\rho}}{d t}=\frac{1}{2} \sum_{j, k}^{2,2} \varepsilon_{j k}\left\{\left[\hat{a}_{j}^{\dagger} \hat{a}_{k}^{\dagger}-\hat{a}_{j} \hat{a}_{k}, \hat{\rho}\right]\right\}+\frac{1}{2} \kappa \sum_{j}^{2}\left\{\left[\hat{a}_{j}, \hat{\rho} \hat{a}_{j}^{\dagger}\right]+\left[\hat{a}_{j} \hat{\rho}, \hat{a}_{j}^{\dagger}\right]\right\},
$$

where $\kappa$ is the coupling constant for the interaction between the cavity and reservoir modes.

\subsection{Cavity Modes}

In this section we seek to find the expression for the cavity mode operators at steady state. With the aid of Eq. (2) and

$$
\frac{d}{d t}\langle\hat{A}(t)\rangle=\operatorname{Tr}\left(\frac{d \hat{\rho}(t)}{d t} \hat{A}\right),
$$

along with the cyclic property of the trace operation and the commutation relations: $\left[\hat{a}_{p}, \hat{a}_{q}^{+}\right]=\delta_{p q},\left[\hat{a}_{p}, \hat{a}_{q}\right]=$ 0 , with $p, q=1,2$, we get

$$
\begin{gathered}
\frac{d\left\langle\hat{a}_{p}\right\rangle}{d t}=-\frac{1}{2} \kappa\left\langle\hat{a}_{p}\right\rangle+\sum_{k=1}^{2} \varepsilon_{p k}\left\langle\hat{a}_{k}^{\dagger}\right\rangle, \\
\frac{d\left\langle\hat{a}_{p} \hat{a}_{q}\right\rangle}{d t}=-\kappa\left\langle\hat{a}_{p} \hat{a}_{q}\right\rangle+\sum_{k=1}^{2} \varepsilon_{q k}\left\langle\hat{a}_{k}^{\dagger} \hat{a}_{p}\right\rangle+\varepsilon_{p k}\left\langle\hat{a}_{k}^{\dagger} \hat{a}_{q}\right\rangle+\varepsilon_{p q}, \\
\frac{d\left\langle\hat{a}_{p}^{\dagger} \hat{a}_{q}\right\rangle}{d t}=-\kappa\left\langle\hat{a}_{p}^{\dagger} \hat{a}_{q}\right\rangle+\sum_{k=1}^{2} \varepsilon_{q k}\left\langle\hat{a}_{k}^{\dagger} \hat{a}_{p}^{\dagger}\right\rangle+\varepsilon_{p k}\left\langle\hat{a}_{k} \hat{a}_{q}\right\rangle .
\end{gathered}
$$

On the bases of Eq. (4), we write

$$
\frac{d \hat{a}_{p}(t)}{d t}=-\frac{1}{2} \sum_{k=1}^{2}\left(\kappa \hat{a}_{k}(t) \delta_{p k}-2 \varepsilon_{p k} \hat{a}_{k}^{\dagger}(t)\right)+\hat{F}_{p}(t),
$$

where $\widehat{F}_{p} s$ are the noise operators having the following correlation properties:

$$
\begin{aligned}
\left\langle\hat{F}_{p}^{\dagger}(t) \hat{F}_{q}\left(t^{\prime}\right)\right\rangle & =\left\langle\hat{F}_{p}\right\rangle=0, \\
\left\langle\hat{F}_{p}(t) \hat{F}_{q}\left(t^{\prime}\right)\right\rangle & =\varepsilon_{p q} \delta\left(t-t^{\prime}\right) .
\end{aligned}
$$

With the aid of Eq. (7) and the definition of quadrature operators for a single-mode light given by

$$
\hat{a}_{p \pm}=\sqrt{ \pm 1}\left(\hat{a}_{p}^{\dagger} \pm \hat{a}_{p}\right),
$$

we can write as

$$
\begin{aligned}
& \hat{a}_{ \pm}(t)=\left(\begin{array}{c}
\hat{a}_{1 \pm}(t) \\
\hat{a}_{2 \pm}(t)
\end{array}\right), \\
& M_{ \pm}=\left(\begin{array}{cc}
\mu_{1 \mp} & \mp 2 \varepsilon_{12} \\
\mp 2 \varepsilon_{12} & \mu_{2 \mp}
\end{array}\right), \\
& \hat{F}_{ \pm}(t)=\left(\begin{array}{c}
\hat{F}_{1 \pm}(t) \\
\hat{F}_{2 \pm}(t)
\end{array}\right),
\end{aligned}
$$

with

$$
\begin{aligned}
& \mu_{p \mp}=\kappa \mp 2 \varepsilon_{p p}, \\
& \hat{F}_{p \pm}=\sqrt{ \pm 1}\left(\hat{F}_{p}^{\dagger} \pm \hat{F}_{p}\right) .
\end{aligned}
$$

We next proceed to seek the steady state solutions of Eq. (9). To this end, introducing a unitary matrix defined by

$$
V=\left(\begin{array}{ll}
v_{11} & v_{12} \\
v_{21} & v_{22}
\end{array}\right)
$$

with $V_{1}=\left(\begin{array}{l}v_{11} \\ v_{21}\end{array}\right)$ and $V_{2}=\left(\begin{array}{l}v_{21} \\ v_{22}\end{array}\right)$ being the eigenvectors of the matrix $M_{ \pm}$, we write

$$
\frac{d}{d t} V^{-1} \hat{a}_{ \pm}(t)=-\frac{1}{2} R_{ \pm} V^{-1} \hat{a}_{ \pm}(t)+V^{-1} \hat{F}_{ \pm}(t),
$$


where

$$
R_{ \pm}=V^{-1} M_{ \pm} V=\left(\begin{array}{cc}
\lambda_{1 \pm} & 0 \\
0 & \lambda_{2 \pm}
\end{array}\right)
$$

and $\lambda_{p \pm}$ are the eigenvalues of $\mathrm{M} \pm$. We note that Eq. (17) will have well defined solutions if both $\lambda_{p \pm}>0$. Hence the solution of Eq. (17) for $t \rightarrow \infty$ can be written as

$$
\hat{a}_{ \pm}=\int_{0}^{t=\infty} V e^{-\frac{1}{2} R_{ \pm}\left(t-t^{\prime}\right)} V^{-1} \hat{F}_{ \pm}\left(t^{\prime}\right) d t^{\prime} .
$$

We next seek the eigenvalues and eigenvectors of $M_{ \pm}$. Applying the eigenvalue equation:

$$
M_{ \pm} V_{p}=\lambda_{ \pm} \bar{V}_{p}
$$

along with Eq (12), we have the characteristic equation:

$$
\lambda_{ \pm}^{2}-\lambda_{ \pm}\left(\mu_{1 \mp}+\mu_{2 \mp}\right)+\mu_{1 \mp} \mu_{2 \mp}-4 \varepsilon_{12}^{2}=0
$$

and the roots of this quadratic equation are

$$
\begin{aligned}
& \lambda_{1 \pm}=\frac{1}{2}\left(\mu_{1 \mp}+\mu_{2 \mp}+\Omega_{\mp}\right), \\
& \lambda_{2 \pm}=\frac{1}{2}\left(\mu_{1 \mp}+\mu_{2 \mp}-\Omega_{\mp}\right),
\end{aligned}
$$

where

$$
\Omega_{\mp}=\sqrt{\left(\mu_{1 \mp}-\mu_{2 \mp}\right)^{2}+16 \varepsilon_{12}^{2}} .
$$

Furthermore, in view of Eqs. (11) and (22), we see that

$$
A_{1 \pm} v_{11} \mp 4 \varepsilon_{12} v_{21}=0 \text {, }
$$

in which

$$
A_{1 \pm}=\mu_{1 \mp}-\mu_{2 \mp}-\Omega_{\mp} .
$$

Taking into account the normalization condition:

$$
v_{11}^{2}+v_{21}^{2}=1
$$

we get

$$
v_{11}= \pm \frac{4 \varepsilon_{12}}{\sqrt{A_{1 \pm}^{2}+16 \varepsilon_{12}^{2}}}, \quad v_{21}=\frac{A_{1 \pm}}{\sqrt{A_{1 \pm}^{2}+16 \varepsilon_{12}^{2}}} .
$$

Similarly the elements of the eigenvector corresponding to $\lambda_{2 \pm}$ is found to be

$$
v_{12}= \pm \frac{4 \varepsilon_{12}}{\sqrt{A_{2 \pm}^{2}+16 \varepsilon_{12}^{2}}}, \quad v_{22}=\frac{A_{2 \pm}}{\sqrt{A_{2 \pm}^{2}+16 \varepsilon_{12}^{2}}},
$$

where

$$
A_{2 \pm}=\mu_{1 \mp}-\mu_{2 \mp}+\Omega_{\mp} .
$$

Substituting Eqs. (28) and (29) into Eq. (16), we see that

$$
V=\left(\begin{array}{cc} 
\pm \frac{2 \varepsilon_{12}}{\sqrt{A_{1 \pm}^{2}+16 \varepsilon_{12}^{2}}} & \pm \frac{4 \varepsilon_{12}}{\sqrt{A_{2 \pm}^{2}+16 \varepsilon_{12}^{2}}} \\
\frac{A_{1 \mp}}{\sqrt{A_{1 \pm}^{2}+16 \varepsilon_{12}^{2}}} & \frac{A_{2 \pm}}{\sqrt{A_{2 \pm}^{2}+16 \varepsilon_{12}^{2}}}
\end{array}\right) .
$$

Taking into account $R_{ \pm}\left(t-t^{\prime}\right)$ is a diagonal matrix and employing Eq. (31) along with the inverse of this equation, we find

$$
V e^{-\frac{1}{2} R_{ \pm}\left(t-t^{\prime}\right)} V^{-1}=\left(\begin{array}{cc}
P_{1 \pm}\left(t-t^{\prime}\right) & Q_{ \pm}\left(t-t^{\prime}\right) \\
Q_{ \pm}\left(t-t^{\prime}\right) & P_{2 \pm}\left(t-t^{\prime}\right)
\end{array}\right),
$$

where

$$
\begin{aligned}
& P_{1 \pm}\left(t-t^{\prime}\right)=\frac{A_{2 \pm}}{2 \Omega_{\mp}} e^{-\frac{1}{2} \lambda_{1 \pm}\left(t-t^{\prime}\right)}-\frac{A_{1 \pm}}{2 \Omega_{\mp}} e^{-\frac{1}{2} \lambda_{2 \pm}\left(t-t^{\prime}\right)}, \\
& P_{2 \pm}\left(t-t^{\prime}\right)=\frac{A_{2 \pm}}{2 \Omega_{\mp}} e^{-\frac{1}{2} \lambda_{2 \pm}\left(t-t^{\prime}\right)}-\frac{A_{1 \pm}}{2 \Omega_{\mp}} e^{-\frac{1}{2} \lambda_{1 \pm}\left(t-t^{\prime}\right)}, \\
& Q_{ \pm}\left(t-t^{\prime}\right)= \pm \frac{4 \varepsilon_{12}}{2 \Omega_{\mp}} e^{-\frac{1}{2} \lambda_{2 \pm}\left(t-t^{\prime}\right)} \mp \frac{4 \varepsilon_{12}}{2 \Omega_{\mp}} e^{-\frac{1}{2} \lambda_{1 \pm}\left(t-t^{\prime}\right)} .
\end{aligned}
$$


With the aid of Eqs. (11), (19), and (32), the quadrature operators can be expressed as

$$
\begin{aligned}
& \hat{a}_{1 \pm}=\int_{0}^{t=\infty}\left(P_{1 \pm}\left(t-t^{\prime}\right) \hat{F}_{1 \pm}\left(t^{\prime}\right)+Q_{ \pm}\left(t-t^{\prime}\right) \hat{F}_{2 \pm}\left(t^{\prime}\right)\right) d t^{\prime} \\
& \hat{a}_{2 \pm}=\int_{0}^{t=\infty}\left(P_{2 \pm}\left(t-t^{\prime}\right) \hat{F}_{2 \pm}\left(t^{\prime}\right)+Q_{ \pm}\left(t-t^{\prime}\right) \hat{F}_{1 \pm}\left(t^{\prime}\right)\right) d t^{\prime} .
\end{aligned}
$$

\section{Quadrature Squeezing}

In this section we seek to calculate the quadrature variances and analyze the effect of single-signal and single-idler light modes on quadrature squeezing property of the two-mode light in the cavity. The squeezing properties of the two-mode light can be described by two quadrature operators defined by

$$
\hat{c}_{ \pm}=\hat{a}_{1 \pm}+\hat{a}_{2 \pm}
$$

with $\hat{a}_{i \pm}$ being quadrature operators defined in Eq. (9). These quadrature operators satisfy the commutation relation

$$
\left[\hat{c}_{+}, \hat{c}_{-}\right]=4 i
$$

On the basis of these, we use a modified criteria that a two-mode light is said to be in squeezed state if either $\Delta c_{+}^{2}<2$ or $\Delta c_{-}^{2}<2$ providing that $\Delta c_{+}^{2} \Delta c_{+}^{2} \geq 4$. Using Eq. (35), the variances of the quadrature operators defined by

$$
\Delta c_{ \pm}^{2}=\left\langle\hat{c}_{ \pm}, \hat{c}_{ \pm}\right\rangle
$$

can be expressed as

$$
\Delta c_{ \pm}^{2}=2+\sum_{p, q}^{2}\left\langle: \hat{a}_{p \pm}, \hat{a}_{q \pm}:\right\rangle,
$$

in which :: stands for the operators in normal order. With the aid of Eqs. (8) and (15), we easily verify that

$$
\begin{aligned}
\left\langle\hat{F}_{p \pm}(t)\right\rangle & =0, \\
\left\langle\hat{F}_{p \pm}(t) \hat{F}_{q \pm}\left(t^{\prime}\right)\right\rangle & = \pm 2 \varepsilon_{p q} \delta\left(t-t^{\prime}\right) .
\end{aligned}
$$

Moreover using Eqs. (14), (34), and (39), we find

$$
\begin{aligned}
& \left\langle: \hat{a}_{1 \pm}, \hat{a}_{1 \pm}:\right\rangle= \pm \frac{2 \varepsilon_{11}\left(\kappa \mp 2 \varepsilon_{22}\right) \pm 4 \varepsilon_{12}^{2}}{\left(\kappa \mp 2 \varepsilon_{11}\right)\left(\kappa \mp 2 \varepsilon_{22}\right)-4 \varepsilon_{12}^{2}}, \\
& \left\langle: \hat{a}_{2 \pm}, \hat{a}_{2 \pm}:\right\rangle= \pm \frac{2 \varepsilon_{22}\left(\kappa \mp 2 \varepsilon_{11}\right) \pm 4 \varepsilon_{12}^{2}}{\left(\kappa \mp 2 \varepsilon_{11}\right)\left(\kappa \mp 2 \varepsilon_{22}\right)-4 \varepsilon_{12}^{2}}, \\
& \left\langle: \hat{a}_{1 \pm}, \hat{a}_{2 \pm}:\right\rangle= \pm \frac{2 \varepsilon_{12} \kappa}{\left(\kappa \mp 2 \varepsilon_{11}\right)\left(\kappa \mp 2 \varepsilon_{22}\right)-4 \varepsilon_{12}^{2}} .
\end{aligned}
$$

Finally, applying Eqs. (40), (41), and (42) in Eq. (38), we find

$$
\Delta c_{ \pm}^{2}=\frac{2 \kappa\left(\kappa \mp \varepsilon_{11} \mp \varepsilon_{22} \pm 2 \varepsilon_{12}\right)}{\left(\kappa \mp 2 \varepsilon_{11}\right)\left(\kappa \mp 2 \varepsilon_{22}\right)-4 \varepsilon_{12}^{2}} \text {. }
$$

Eq. (43) represents the quadrature variances for two-mode light produced by the combination of degenerate and nondegenerate parametric oscillators.

It is interesting to consider some special cases for the system. For the case in the absence of nonlinear crystals that produce signal-signal modes, the system reduces to non-degenerate parametric oscillator. Thus upon setting $\varepsilon_{11}=\varepsilon_{22}=0$ in Eq. (43), the quadrature variances take the form

$$
\Delta c_{ \pm}^{2}=\frac{2 \kappa}{\kappa \mp 2 \varepsilon_{12}} .
$$

We see from Eq. (44) that nondegenerate parametric oscillator produces light in squeezed state and the squeezing occurs in the minus quadrature. Moreover, It is also possible to obtain $50 \%$ squeezing on the minus quadrature at critical point $\kappa=2 \varepsilon_{12}$.

For the case in the absence of nonlinear crystal that generates single-idler modes, we set $\varepsilon_{12}=0$ in Eq. (43), so that the system reduces to the quadrature variances of two-mode light produced by two degenerate parametric oscillators:

$$
\Delta c_{ \pm}^{2}=\frac{2 \kappa\left(\kappa \mp \varepsilon_{11} \mp \varepsilon_{22}\right)}{\left(\kappa \mp 2 \varepsilon_{11}\right)\left(\kappa \mp 2 \varepsilon_{22}\right)} .
$$


Eq. (45) shows that the two-mode light produced by two degenerate parametric oscillators are in squeezed state. If the amplitudes proportional to the pump modes are taken to be equal, the quadrature variances of the two-mode light produced by two degenerate parametric oscillators will have same forms as that of the nondegenerate parametric oscillator.

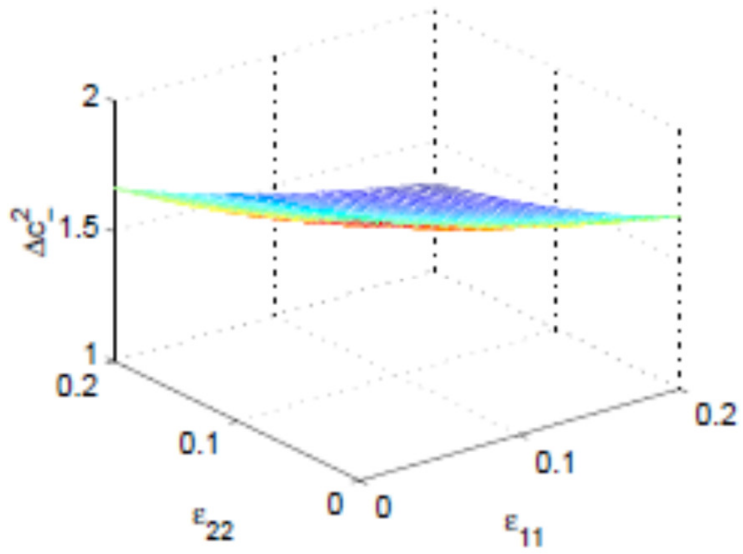

a. $\varepsilon_{12}=0.00$

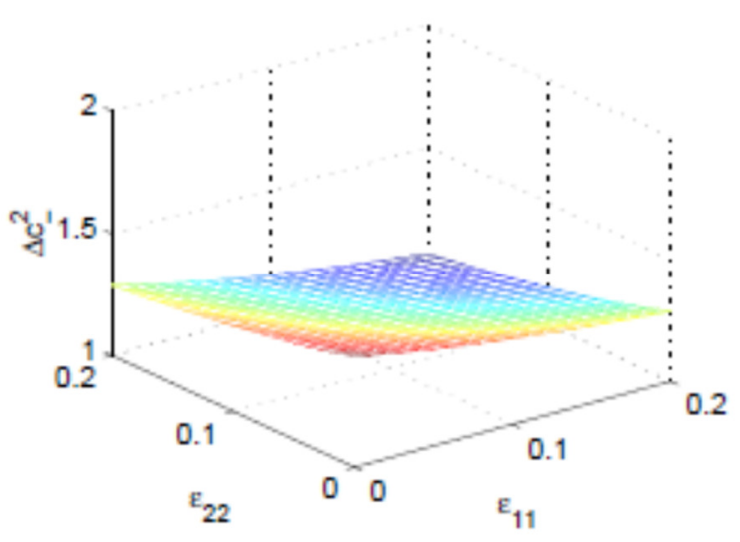

c. $\varepsilon_{12}=0.15$

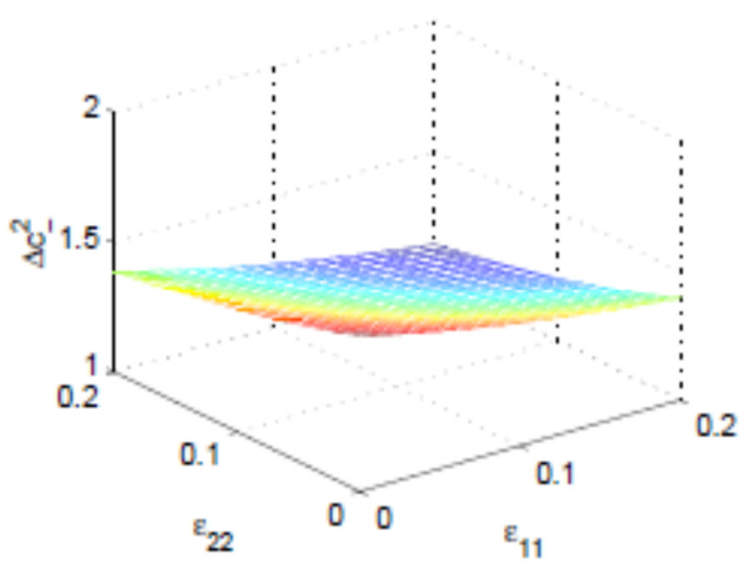

b. $\varepsilon_{12}=0.10$

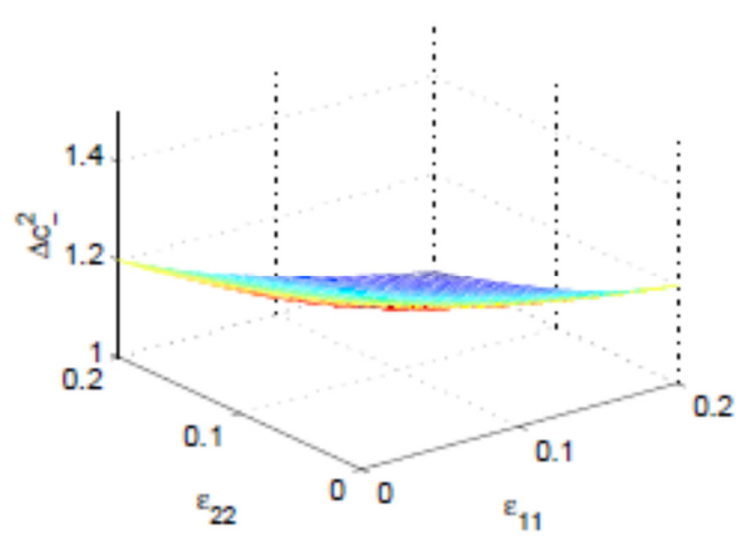

d. $\varepsilon_{12}=0.20$

FIG. 2: The plots of the minus quadrature variances versus $\varepsilon_{11}$ and $\varepsilon_{22}$ for $\kappa=0.8$ and for different values of $\varepsilon_{12}$.

In FIG. 2 we plot the variance of the minus quadrature [Eq. (43)] versus $\varepsilon_{11}$ and $\varepsilon_{22}$ for $\kappa=0.8$ and for different values of the amplitude proportional to pump mode of the nondegenerate parametric oscillator, for $\varepsilon_{12}=$ 0 (FIG. 2a), $\varepsilon_{12}=0.10$ (FIG. 2b), $\varepsilon_{12}=0.15$ (FIG. 2c), and $\varepsilon_{12}=0.20$. (FIG. 2d). We note from these figures that the two-mode light generated by the system under consideration exhibits squeezing in the minus quadrature and the degree of squeezing increases with the amplitudes proportional to the pump modes. The figures also show that the degree of squeezing is relatively better where $\varepsilon_{11}=\varepsilon_{22}$ compared to $\varepsilon_{11} \neq \varepsilon_{22}$.

We now consider the cases at a critical point $\varepsilon_{12}=\sqrt{\left(\kappa-2 \varepsilon_{11}\right)\left(\kappa-2 \varepsilon_{22}\right)} / 2$. At this point the quadrature of the plus quadrature blows up and the minus becomes

$$
\Delta c_{-}^{2}=\frac{\kappa+\varepsilon_{11}+\varepsilon_{22}-\sqrt{\left(\kappa-2 \varepsilon_{11}\right)\left(\kappa-2 \varepsilon_{22}\right)}}{2\left(\varepsilon_{11}+\varepsilon_{22}\right)} .
$$

Furthermore setting $\varepsilon_{11}=\varepsilon_{22}$ in Eq. (46), we have $\Delta c_{-}^{2}=1$. It implies that $50 \%$ of noise reduction can be obtained at critical points with $\varepsilon_{11}=\varepsilon_{22}$. 


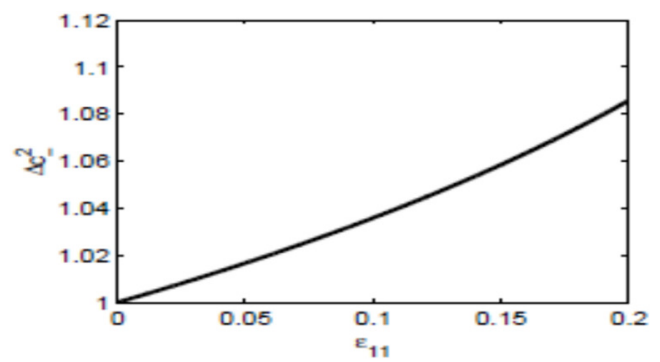

a. $\varepsilon_{22}=0.00$

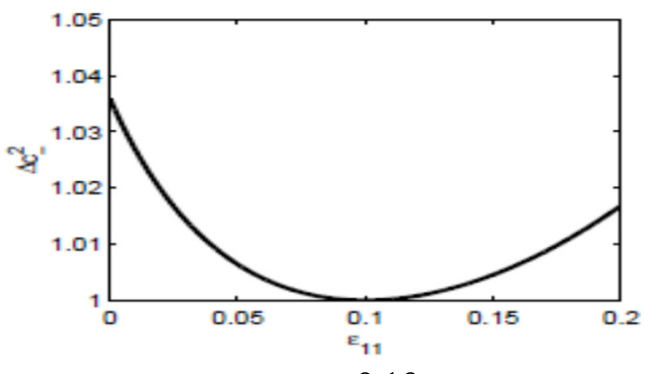

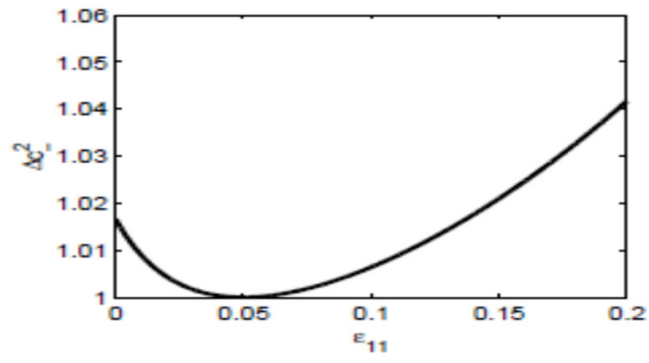

b. $\varepsilon_{22}=0.05$

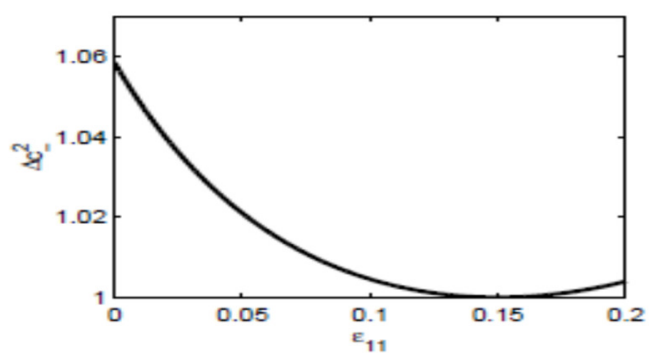

d. $\varepsilon_{22}=0.15$

FIG. 3: The plots of the minus quadrature variance versus $\varepsilon_{11}$ for $\kappa=0.8$ and different values of $\varepsilon_{22}$ at critical points.

FIG. 3 represents the plots of the variance of the minus quadrature [Eq. (46)] versus $\varepsilon_{11}$ for $\kappa=0.8$ and for different values of $\varepsilon_{22}=0.00$ (FIG. 3a), $\varepsilon_{22}=0.05$ (FIG. 3b), $\varepsilon_{22}=0.10$ (FIG. 3c), and $\varepsilon_{22}=0.15$ (FIG. 3d). The plots in FIG. 3 indicate that the degree of squeezing increases as the difference between $\varepsilon_{11}$ and $\varepsilon_{22}$ decreases.

\section{Entanglement}

The entanglement between the modes is well established by Duan et al. [12]. Defining the combined quadratures $\hat{u}=\hat{a}_{1+}-\hat{a}_{2+}$ and $\hat{v}=\hat{a}_{1-}+\hat{a}_{2-}$, a system is said to be entangled if the sum of the variances of the two EPRlike operators of the two modes satisfy the inequality:

$$
\Delta u^{2}+\Delta v^{2}<4
$$

It can be readily established that the steady state variances of these operators in terms of anhilation and creation cavity mode operators to be

$$
\Delta u^{2}+\Delta v^{2}=\Delta c_{+}^{2}+\Delta c_{-}^{2}-4\left\langle\hat{a}_{1+}, \hat{a}_{2+}\right\rangle .
$$

Taking into account Eq. (42) along with Eq. (43), the sum of $\Delta u^{2}$ and $\Delta v^{2}$ can be expressed as

$$
\Delta u^{2}+\Delta v^{2}=\frac{2 \kappa\left(\kappa-\varepsilon_{11}-\varepsilon_{22}-2 \varepsilon_{12}\right)}{\left(\kappa-2 \varepsilon_{11}\right)\left(\kappa-2 \varepsilon_{22}\right)-4 \varepsilon_{12}^{2}}+\frac{2 \kappa\left(\kappa+\varepsilon_{11}+\varepsilon_{22}-2 \varepsilon_{12}\right)}{\left(\kappa+2 \varepsilon_{11}\right)\left(\kappa+2 \varepsilon_{22}\right)-4 \varepsilon_{12}^{2}} \text {. }
$$




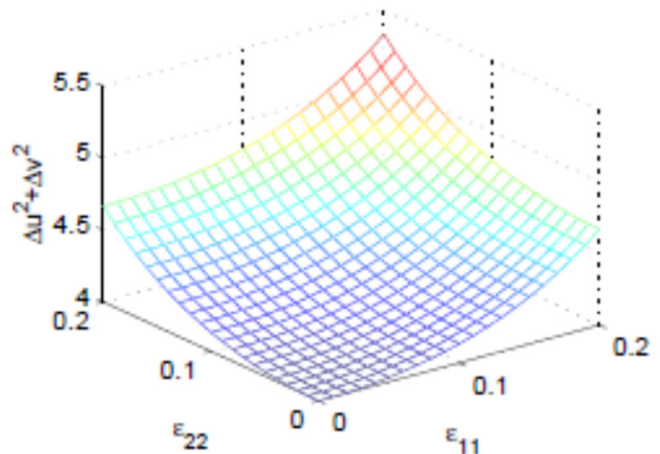

a. $\varepsilon_{12}=0.00$

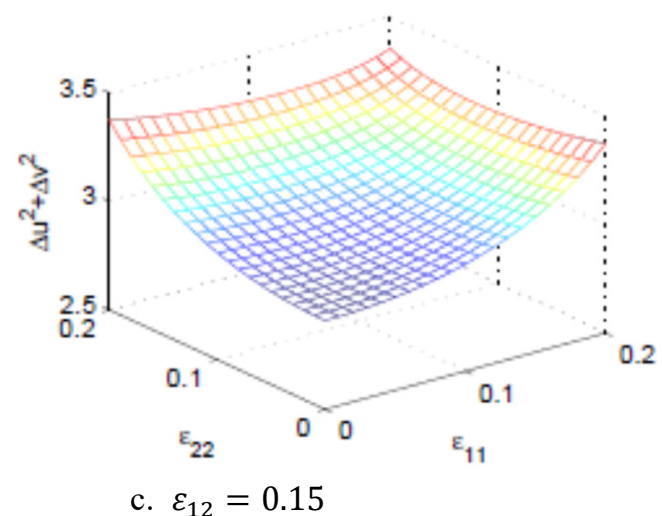

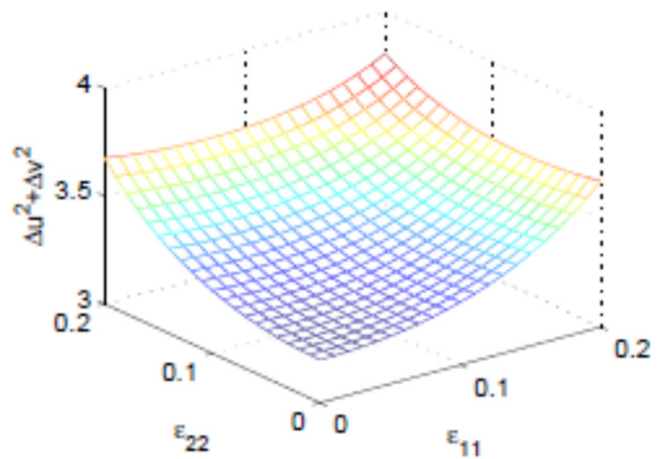

b. $\varepsilon_{12}=0.10$

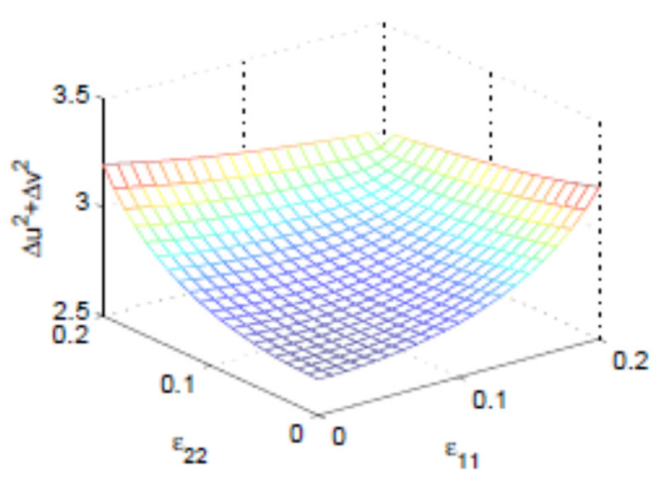

d. $\varepsilon_{12}=0.20$

FIG. 4: The plots of $\Delta u^{2}+\Delta v^{2}$ versus $\varepsilon_{11}$ and $\varepsilon_{22}$ for $\kappa=0.8$ and for different values of $\varepsilon_{12}$.

In FIG. 4 we have plotted the sum of $\Delta u^{2}$ and $\Delta v^{2}$ [Eq. (49)] versus $\varepsilon_{11}$ and $\varepsilon_{22}$ for $\kappa=0.8$ and for different values of the amplitude proportional to pump mode $\varepsilon_{12}=0$ (FIG. $4 \mathrm{a}$ ), $\varepsilon_{12}=0.10$ (FIG. $4 \mathrm{~b}$ ), $\varepsilon_{12}=0.15$ (FIG. $4 \mathrm{c}$ ), and $\varepsilon_{12}=0.20$ (FIG. 2d). The plot in FIG. 4a indicates that the two-mode light produced by degenerate parametric oscillators don't exhibit entanglement property this may be due no correlation between the two signal modes. On the other, The other plots show that the signal-idler modes produced by nondegenerate parametric are the source of entanglement properties for the cavity modes produced by the system under consideration and the degree of entanglement increases with the amplitude proportional to the pump mode of the nondegenerate parametric oscillator $\varepsilon_{12}$.

\section{Conclusion}

In this work, we have considered the cavity modes produced by combination of non-degenerate and two degenerate parametric process. Employing the master equation, we have obtained equation of motion for the expectation values of the cavity mode operators. Using the steady state solutions of the resulting equations, we have determined the quadrature variances of the two-mode light. Our analysis showed that the system under consideration can produce a light in squeezed state and the squeezing occurs in the minus quadrature. Moreover, signal-signal and signal-idler modes produced by degenerate and nondegenerate subharmonic generations, respectively enhance the degree of quadrature squeezing. We have also studied the entanglement properties of the two-modes as steady state. The result shows that the entangled light modes are produced by non-degenerate parametric oscillator but the modes produced by degenerate parametric oscillators reduce the entanglement properties of the two-mode light.

\section{References}

[1] K. Fesseha, Opt. Commun. 156, (1998) 145.

[2] N. A. Ansari, Phys. Rev. A 48, (1993) 4686.

[3] P.D. Drummond, M.D. Reid, Phys. Rev. A 41, (1990) 3930.

[4] J. Anwar and M. S. Zubairy, Phys. Rev. A 45, (1992) 1804.

[5] B. Daniel and K. Fesseha, Opt. Commun. 151, (1998) 384.

[6] B. Teklu, Opt. Commun. 261, (2006) 310.

[7] S. Tesfa, Eur. Phys. J D 43, (2008) 3351.

[8] A. Mebrahtu, J. Mod. Opt 52, (2005) 813.

[9] M. J. Collett and C. W. Gardiner, Phys. Rev. A 30, (1984) 1386.

[10] L. A. Lugiato and G. Strini, Opt. Commun. 41, (1982) 67. 
[11] H. Deng, D. Erenso, R. Vyas, and S. Singh, Phys. Rev. Lett. 86, (2001) 2770.

[12] G. J. Milburn and D. F. Walls, Opt. Commun. 39, (1981) 401.

[13] J. Anwar and M. S. Zubairy, Phys. Rev. A 49, (1994) 481.

[14] M.D. Reid, P.D. Drummond, Phys. Rev. Lett. 60, (1988) 2731.

[15] S. Lloyd, S.L. Braunstein, Phys. Rev. Lett. 82, (1999) 1784.

[16] T.C. Ralph, Phys.Rev. A 61, (2000) 010302.

[17] K. Dechoum, et al., Phys. Rev. Lett. A70, (2004) 053807.

[18] Y. Zhang, H. Wang, X. Li, J. Jing, C. Xie, K. Peng, Phys. Rev. A 62, (2000) 023813.

[19] L.-M. Duan, G. Giedke, J. I. Cirac, and P. Zoller, Phys. Rev. Lett. 84, (2000) 2722. 